\title{
Formation of InN nanoparticle and nanorod structures by nitrogen plasma annealing method
}

\author{
AJAY KUMAR MANN, DEEPAK VARANDANI, BODH RAJ MEHTA*, \\ LALIT KUMAR MALHOTRA, G MANGAMMA ${ }^{\dagger}$ and A K TYAGI ${ }^{\dagger}$ \\ Department of Physics, Indian Institute of Technology Delhi, New Delhi 110 016, India \\ ${ }^{\dagger}$ Material Science Division, Indira Gandhi Centre for Atomic Research, Kalpakkam 603 102, India
}

\begin{abstract}
In the present study, a novel method involving nitrogen plasma annealing has been reported for preparing InN nanoparticle/nanorod structures and for improving the properties of InN nanoparticle layers. Plasma annealed structures have been characterized by $X$-ray diffraction, atomic force microscopy and photoluminescence spectroscopy techniques. InN nanoparticle layers have been prepared using activated reactive evaporation set up. It has been observed that there is a remarkable improvement in the conductivity and crystallinity of InN nanoparticle layers on annealing in nitrogen plasma. This has been attributed to the increase in the nitrogen content of the samples. Experiments involving plasma annealing of In nanorods deposited oxide template has also been carried out. It was found that on plasma treatment In nanorods get converted to mixed phase InN nanorods with hexagonal and cubic fractions.
\end{abstract}

Keywords. Indium; indium nitride; plasma annealing.

\section{Introduction}

Indium nitride is a well known III-V semiconductor with potential applications in the field of electronics and optoelectronics (Butcher and Tansley 2005). It has the lowest effective mass (Fu and Chen 2004) among its analogous class of materials and consequently a high drift velocity, and thus superior electron transport properties. InN on alloying with high bandgap materials (such as GaN and AIN) can have possible emission ranging from infrared to ultraviolet region of the electromagnetic spectrum. Therefore, it can be used as active material in photonic devices such as lasers and light emitting diodes (Mahboob et al 2004). It crystallizes into two different structures, the hexagonal (wurtzite) which is its stable form and cubic (zinc-blende) which is the metastable form. Most of the synthesis techniques report the formation of hexagonal phase. Cubic phase being the metastable phase is not formed in general equilibrium conditions. However, it is quite useful due to its application as active layer in optoelectronic devices (Chai et al 2007).

The bandgap value of InN has been a topic of contention for some time now and different values ranging from $0.7-2.2 \mathrm{eV}$ have been reported (Tansley et al 1986; Inushima et al 2001; Davydov et al 2002; Wu et al 2004). A number of reasons have been put forward by various researchers which could lead to such large variations in the bandgap. These include presence of oxygen impurities (Butcher and Tansley 2005), Burstein Moss shift (Wu

*Author for correspondence (brmehta@physics.iitd.ernet.in) et al 2004), defect levels (Bhuiyan et al 2003) and particle size effects (Xiao et al 2003). Indium nitride has a relatively low dissociation temperature of around $500^{\circ} \mathrm{C}$ (Bhuiyan et al 2003), which renders synthesis of high quality samples a difficult proposition. The major drawbacks of majority of the reported techniques used for synthesis of InN are the use of high temperatures ( $\mathrm{Wu}$ et al 2002), or corrosive gases (Koukitu et al 1997) such as ammonia. High temperature synthesis leads to the presence of defects while use of ammonia as precursor gas is known to cause quenching of the luminescence signals due to $\mathrm{H}$ incorporation (Koukitu et al 1997). Thus, there is an urgent need for novel synthesis techniques which can work in milder conditions, particularly low temperatures and also avoid the use of ammonia. The use of plasma ambient composed of relatively high energy electrons, positive ions and activated neutrals during synthesis or post deposition annealing can serve these objectives. There are not many studies in literature on the effect of nitrogen plasma annealing on the structural and optical properties of indium or indium nitride. This paper reports a series of experiments carried out in this direction. In the first experiment, InN layers were deposited by evaporating In through a nitrogen plasma in an activated reactive evaporation set up. The second experiment is based on studying the effect of nitrogen plasma annealing of InN layers. In addition, plasma annealing of In nanorods electrodeposited inside anodic alumina membrane (AAM) have also been carried out in a separate experiment. For comparison, vacuum evaporated thin films have also been exposed to nitrogen plasma under similar conditions. 


\section{Experimental}

Two different set of samples were synthesized which were subsequently annealed in nitrogen plasma at different temperatures and for varied times to see the effect of annealing on structural, optical and electronic properties. The samples are designated as: A1-A3 and B1-B4, the details of which have been given below.

(a) Sample Al represents $\mathrm{InN}$ nanocrystalline layer formed by activated reactive evaporation (ARE) technique on glass substrate. Samples denoted as A2 and A3 are InN nanocrystalline layers synthesized by ARE technique and subsequently annealed in nitrogen plasma for $1 \mathrm{~h}$ at constant substrate temperatures of room temperature $(R T)$ and $250^{\circ} \mathrm{C}$, respectively. The details of sample preparation are described elsewhere (Mann et al 2005).

(b) Sample B1 represents In nanorods/AAM assembly synthesized by electrochemical deposition and B2 represents the In/AAM sample annealed in nitrogen plasma for $5 \mathrm{~h}$ at $R T$. For electrochemical deposition of indium, commercially available AAM substrates having uniformly distributed nanometer sized pores obtained from Whatman International Limited were used. The AAM was pasted on the fluorine doped tin oxide (FTO) substrates. The deposition was carried out in an electrochemical cell with AAM/FTO assembly acting as the cathode and a platinum strip as the anode. The electrolyte consisted of $10 \mathrm{mM}$ solution of $\mathrm{InCl}_{3} \cdot 3 \mathrm{H}_{2} \mathrm{O}$ in water and $\mathrm{HCl}$ was added to control the $\mathrm{pH}$ value. The deposition was carried out at a current of $500 \mu \mathrm{A}$ leading to formation of indium nanorods inside the AAM pores.

A set of indium thin films deposited by thermal evaporation on glass substrates were also annealed in nitrogen plasma for comparison with the experiment on nanorod structures. Sample B3 represents indium thin films synthesized in a vacuum of the order of $1 \times 10^{-6}$ torr. Sample $\mathrm{B} 4$ was annealed in nitrogen plasma for $3 \mathrm{~h}$ at $R T$.

Table 1 describes the deposition and annealing parameters used in the present study. X-ray diffraction (XRD) measurements on the samples were done using Phillips X'Pert Pro-PW3040 diffractometer. Electron microscopic studies were carried out using transmission electron microscope (TEM) (Philips, Model CM12) operated at $120 \mathrm{kV}$. Photoluminescence (PL) measurements were carried out with an argon ion laser (wavelength, $488 \mathrm{~nm}$ ) as the excitation source. For dispersion and detection of the signal from the sample, spectrometer attached to Scanning Near Field Optical Microscope (SNOM) equipment supplied by NTMDT, Russia, was used. Atomic force microscopic (AFM) measurements were done using Nanoscope IIIa, Veeco Metrology Group equipment. Conducting atomic force microscopic (CAFM) measurements were done in contact mode with images being scanned at several points at sample bias of (-) $100 \mathrm{mV}$. X-ray photoelectron spectroscopy (XPS) analysis was done by using Perkin-Elmer1257 instrument which consists of a $\operatorname{MgK} \alpha$ radiation source (energy $=1253.6 \mathrm{eV}$ ) and a hemispherical section analyser with $25 \mathrm{meV}$ resolution. Optical transmittance and reflectance were measured with UV-vis-NIR spectrophotometer (Perkin Elmer Lambda 900) which has a resolution of around $0 \cdot 1 \mathrm{~nm}$.

\section{Results and discussion}

\subsection{InN nanocrystalline layer deposited by ARE}

(i) Before plasma annealing: In this section structural and surface morphological studies carried out for sample Al are presented and discussed.

3.1a Structural analysis: Figure 1 depicts the XRD pattern of sample Al, which has been deposited using ARE technique without any post-deposition treatment. It is seen that all the peaks are broad which points towards the nanocrystalline nature of deposited layer. The observed ' $d$ ' values match closely with the experimentally reported ' $d$ ' values of hexagonal (wurtzite) indium nitride as indicated in JCPDS file no $02-1450$. The maximum intensity peak occurs at $2 \theta=32.91^{\circ}$ and $d=2.71 \AA$, corresponding to (101) plane. In addition, three weak peaks at $2 \theta=$ $29.05^{\circ} \mathrm{C}$ and $d=3.07 \AA$ corresponding to (100) plane, $2 \theta=43.05^{\circ} \mathrm{C}$ and $d=2.09 \AA$ corresponding to (102) plane and $2 \theta=51.27^{\circ} \mathrm{C}$ and $d=1.78 \AA$ corresponding to (110) plane have been observed. There is no peak corresponding to indium or $\operatorname{In}_{2} \mathrm{O}_{3}$ phases.

3.1b Surface morphology analysis: Figure 2(a) shows the two-dimensional AFM image of sample A1. It is evi-

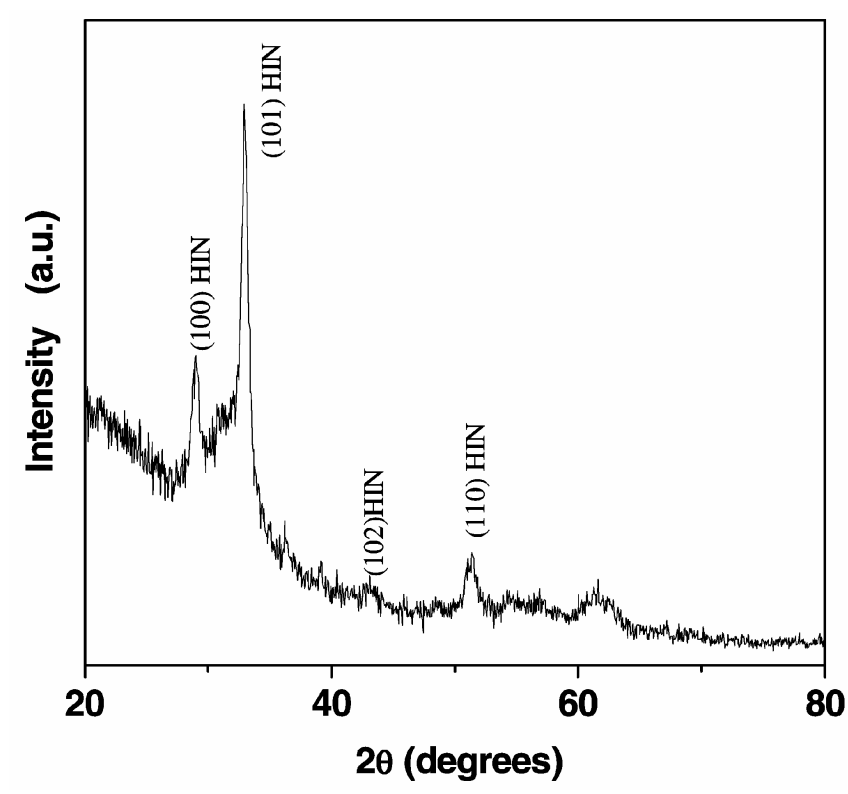

Figure 1. GAXRD of sample Al. The symbol HIN appended to the miller indices is used to indicate that the peaks belong to hexagonal indium nitride. 
Table 1. Details of deposition and annealing parameters.

\begin{tabular}{lllll}
\hline Sample name & Deposition technique & \multicolumn{1}{c}{ Initial phase } & $\begin{array}{c}\text { Post deposition } \\
\text { N-plasma annealing } \\
\text { (temperature) }\left({ }^{\circ} \mathrm{C}\right)\end{array}$ & Final phase \\
\hline A1 & ARE & InN layer & - & - \\
A2 & ARE & Nanocrystalline InN & 30 & Nanocrystalline InN \\
A3 & ARE & Nanocrystalline InN & 250 & Nanocrystalline InN \\
B1 & Electrochemical & In nanorods/AAM & - & - \\
B2 & Electrochemical & In nanorods/AAM & Room temperature & InN nanorods/AAM \\
B3 & Thermal evaporation & In thin film & - & - \\
B4 & Thermal evaporation & In thin film & Room temperature & InN thin film \\
\hline
\end{tabular}
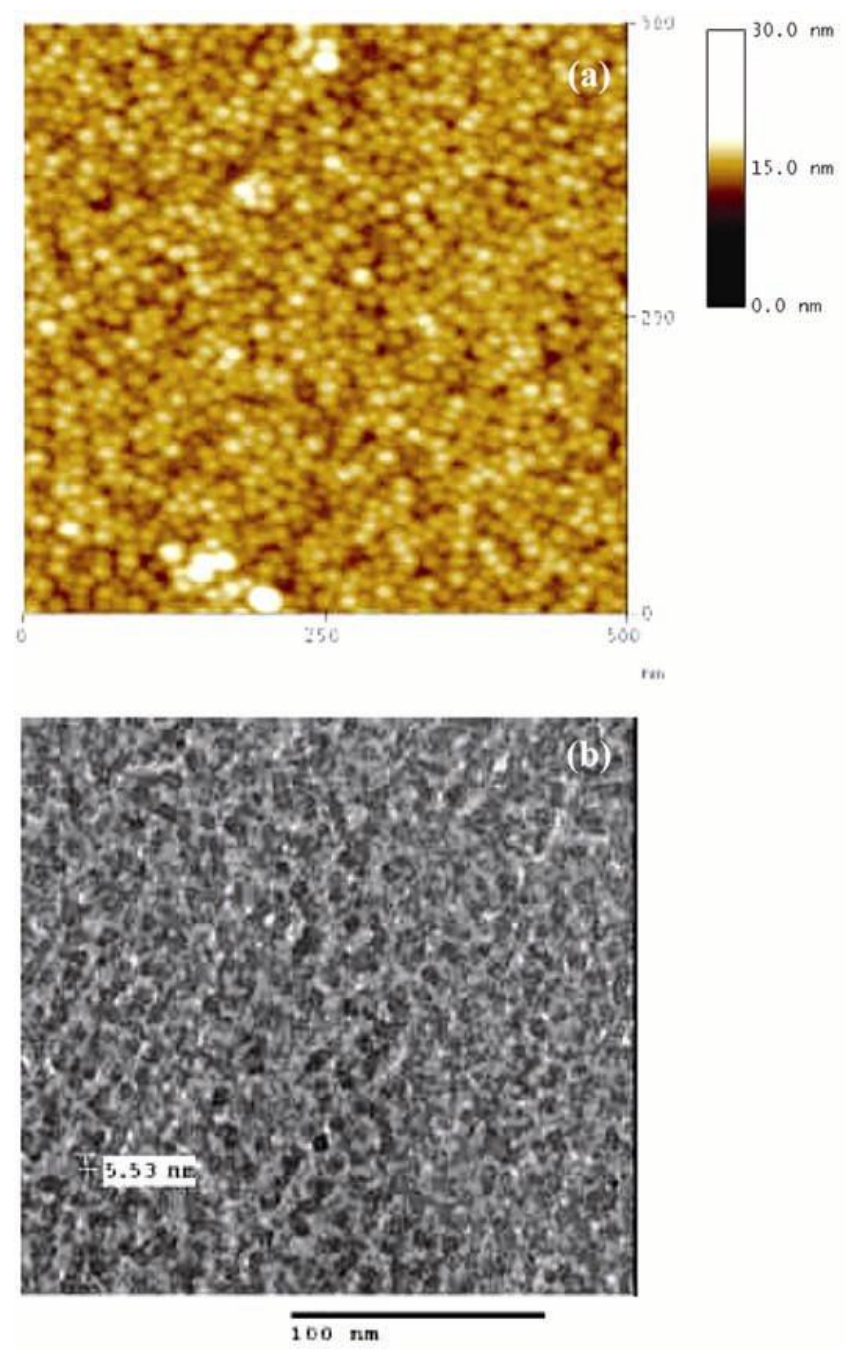

Figure 2. (a) AFM and (b) TEM of sample A1.

dent that the sample comprises of uniformly distributed particles with a small size distribution. The average particle size has been calculated from the AFM image and is around $10 \mathrm{~nm}$. The sample has a reasonably smooth surface with a root mean square roughness of around $1.09 \mathrm{~nm}$. For comparison the TEM image of the sample has been presented in figure 2(b), which shows the uniform cover- age by InN layer. The average particle size calculated from TEM image turns out to be around $6 \mathrm{~nm}$. Thus XRD, AFM and TEM results confirm the nanocrystalline nature of the as-deposited InN layer.

(ii) After plasma annealing: This section deals with the structural and optical absorption measurements on ARE deposited InN nanocrystalline layers (samples A2 and A3), which are subjected to post deposition nitrogen plasma annealing at varying substrate temperatures. In addition, morphological/conductivity measurements using CAFM have also been carried out.

3.1c Structural analysis: Structural characterization of samples A2 and A3 has been carried out by XPS and XRD (not shown here). XRD results show that annealing in nitrogen plasma does not change the already formed wurtzite phase of indium nitride (Mann et al 2007). XPS studies have been carried out to study the electronic properties of sample A3. The measurements have been made after 5-10 min of sputter cleaning to avoid the effect of surface contamination. Carbon and oxygen were found at the surface and after sputter cleaning, peaks corresponding to $\mathrm{C}$ and $\mathrm{O}$ were completely absent. All peak positions have been precisely determined on the basis of $\mathrm{C} 1 s$ peak (at $284.6 \mathrm{eV}$ ) as the reference.

Core level X-ray photoelectron spectra of the sample for $\mathrm{N} 1 s$ peak is shown in figure 3(a). The figure reveals two peaks, a high intensity peak at $397.17 \mathrm{eV}$ and a low intensity peak at $399 \mathrm{eV}$. The peak at $399 \mathrm{eV}$ is due to elemental nitrogen while the peak at $397.17 \mathrm{eV}$ may correspond to the shift in the nitrogen peak as a result of formation of $\mathrm{InN}$. The presence of two peaks shows that small amount of nitrogen in elemental form is present in the sample. Figure 3(b) shows the core level XPS spectra of the sample for In $4 d$ peak. The binding energy peak for In $4 d_{3 / 2}$ was observed at $444.15 \mathrm{eV}$ instead of elemental In $4 d_{3 / 2}$ value of $443.6 \mathrm{eV}$. The binding energy peak position for In $4 d_{5 / 2}$ was observed at $451.81 \mathrm{eV}$ instead of elemental position at $451.20 \mathrm{eV}$. The core level values of In $4 d$ show a positive shift from the elemental indium energy position. Similarly high intensity peak of N $1 s$ 
level has a negative shift from the elemental nitrogen peak value. The shift in binding energy of In $4 d$ and $\mathrm{N} 1 s$ confirm the bonding between In and $\mathrm{N}$ and consequently the formation of InN phase. Also the binding energy values of In $4 d_{3 / 2}$ and $\mathrm{N} 1 s$ matches with the reported values of binding energy for InN (Xiao et al 2003). XPS analysis thus establishes that N-plasma annealing of $\mathrm{InN}$ nanocrystalline layer increases the $\mathrm{N}$-content in them.

3.1d Surface morphological analysis: Figure 4 shows the topographical images (left) and conductance maps (right) for InN nanocrystalline layer samples A2 and A3, respectively. It must be mentioned that there is no oneto-one correspondence between the topographical and conductance images. The average nanoparticle size calculated from conductance maps is 20 and $30 \mathrm{~nm}$ for samples A2 and A3, respectively. In the conductance maps
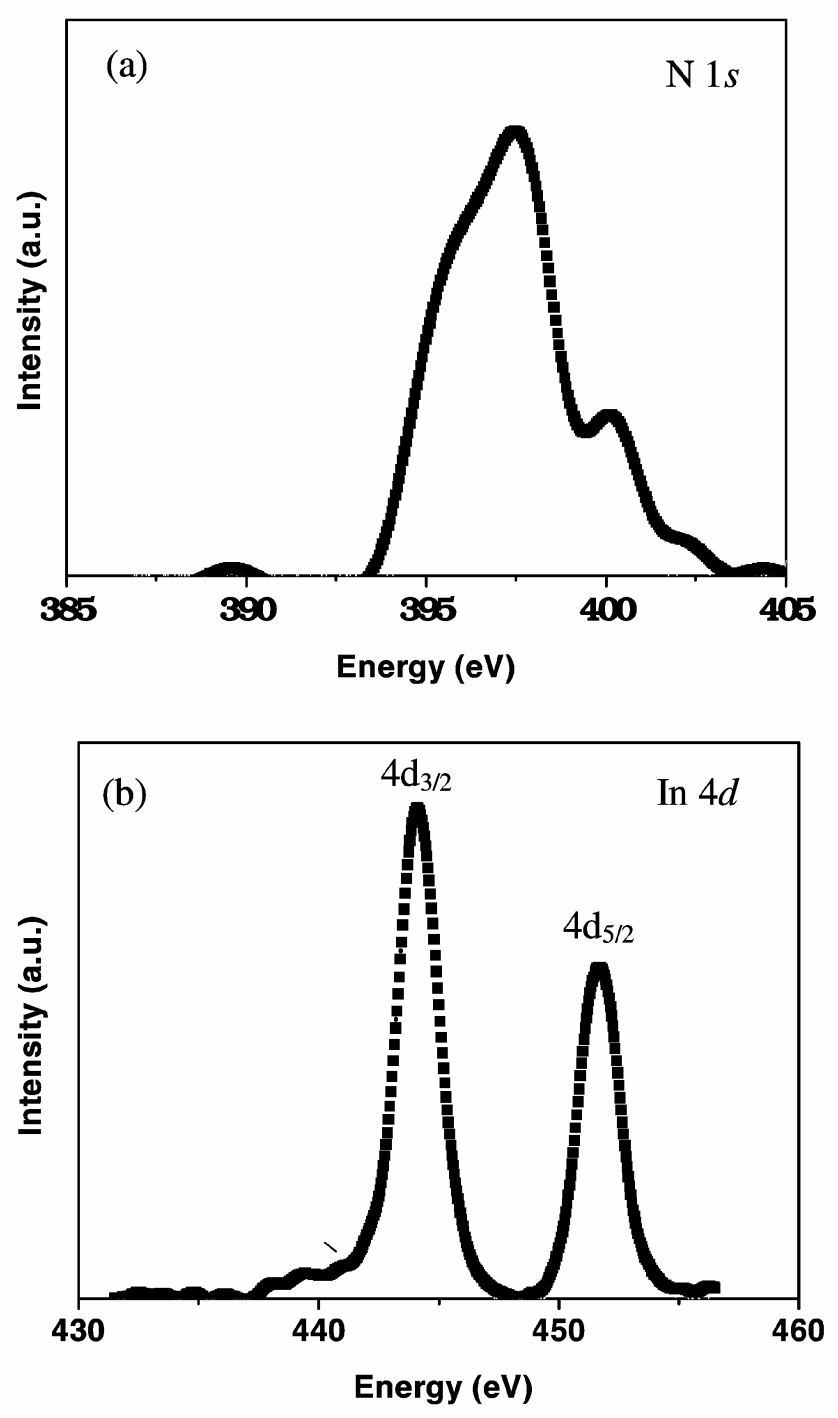

Figure 3. (a) Core level XPS spectra of N1s and (b) core level XPS spectra of In $4 d$ for sample A2. the brighter areas, exhibiting higher currents, represent the crystalline InN phase whereas dark or low brightness areas may correspond to the low conducting, possibly amorphous, InN or $\operatorname{In}_{2} \mathrm{O}_{3}$ phases (Mann et al 2007). Annealing in $\mathrm{N}_{2}$ plasma increases the uniformity in the conductivity of the samples as is evident from conductance images. The usefulness of the conductance maps to clearly identify InN crystallites is quite evident. These features are hard to distinguish in the AFM topographical images. The crystallinity and conductivity of the films increases on plasma annealing which may be due to excess nitrogen in the films which is also evident from the XPS and optical absorption studies (discussed later) of these films. It seems that there is an increased interlinking of conducting regions as the annealing temperature is increased.

3.1e Optical analysis: The transmittance and reflectance spectra of samples A2 and A3 have been obtained by UV-vis-NIR spectroscopy. The absorption coefficient $(\alpha)$ of these samples has been calculated from the reflectance and transmittance data and using their thickness. A plot of $(\alpha h v)^{2}$ vs $h v$ for the samples A2 and A3 is shown in figure 5 . The curve is reasonably constant up to a point and then rises sharply. A linear fit has been carried out in the region where $(\alpha h v)^{2}$ value rises sharply. Absorption edge has been calculated by extrapolating the linear portion of the plot $(\alpha h v)^{2}$ vs $h v$, taking the point where $(\alpha h v)^{2}$ approaches 0 . It has been found that absorption edge increases from $1.48 \mathrm{eV}$ to $1.73 \mathrm{eV}$ for samples A2 to A3. The increase in absorption edge due to quantum confinement may be ruled out as the size of nanoparticles in the present study is large as compared to the Bohr excitonic radius of $\mathrm{InN}$ which has been reported to be in the range of 10-12 $\mathrm{nm}$ (Butcher and Tansley 2005). A possible reason for the observed blue shift may be Burstein moss effect which occurs at high carrier concentrations. Although large carrier concentration may lead to either increase in bandgap due to Burstein moss shift or decrease in its value as a result of bandgap renormalization due to electron-electron interactions. For a low effective mass, semiconductor like InN Burstein moss shift dominates over band renormalization as is also evidenced experimentally in the present case. The carrier concentrations of as deposited and annealed samples are $4 \times 10^{20}$ and $5 \times 10^{21} \mathrm{~cm}^{-3}$, respectively, as reported earlier (Mann et al 2007). It has been shown in earlier reports (Mann et al 2007) that spatial distribution of high conducting regions and their interlinking (CAFM results) modifies the conductivity values of the samples. The conductivity of the as deposited and annealed samples are $4 \times 10^{1}$ and $7.5 \times 10^{3}$ $\left(\Omega^{-1} \mathrm{~cm}^{-1}\right)$, respectively. The expected decrease in the conductivity due to increase in carrier concentration is normally offset by increase in interlinking of high conducting regions which reduces the grain boundary scattering in these samples. These results are in agreement with the 

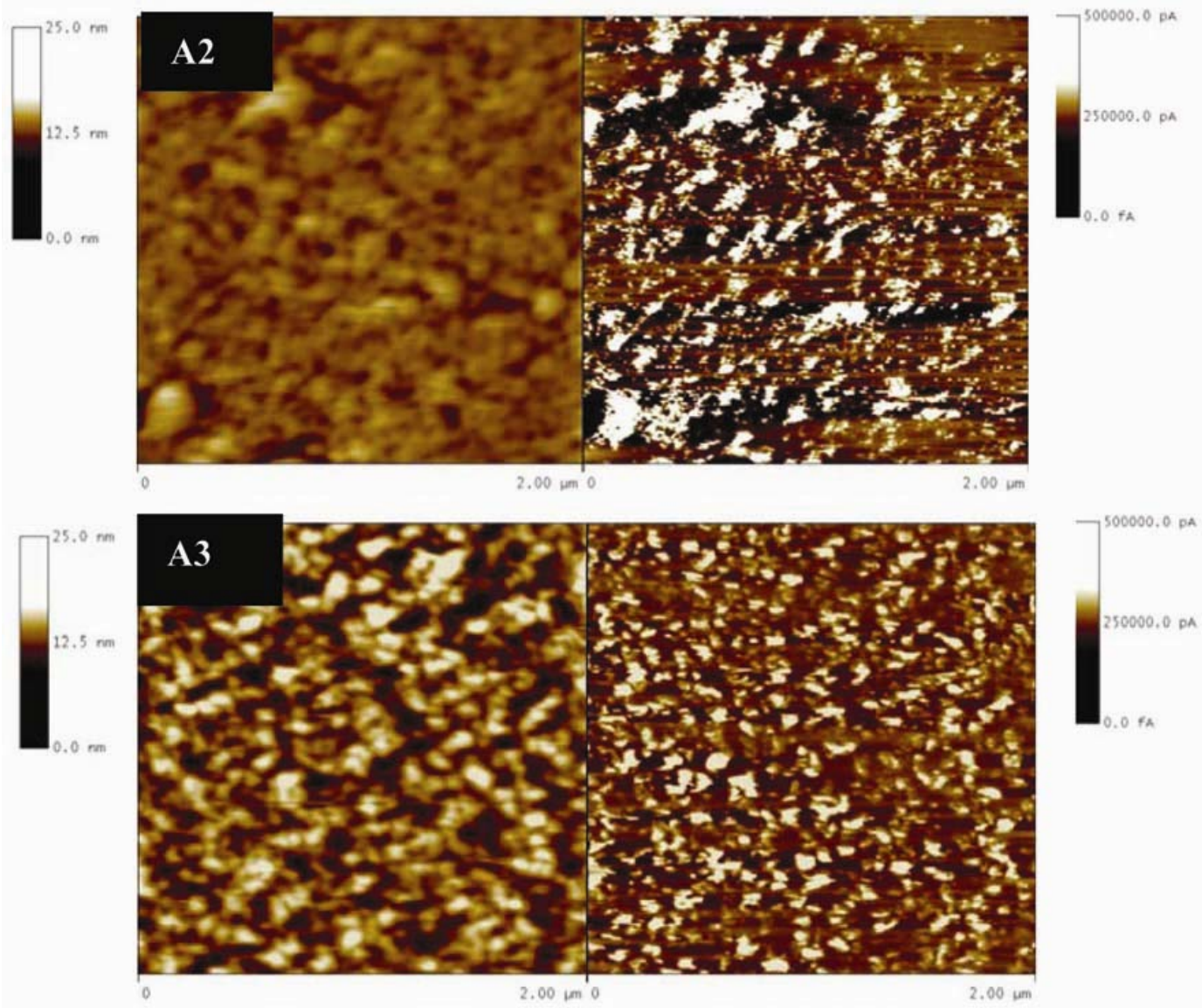

Figure 4. Surface morphology (left) and conductance maps (right) of samples A2 and A3.

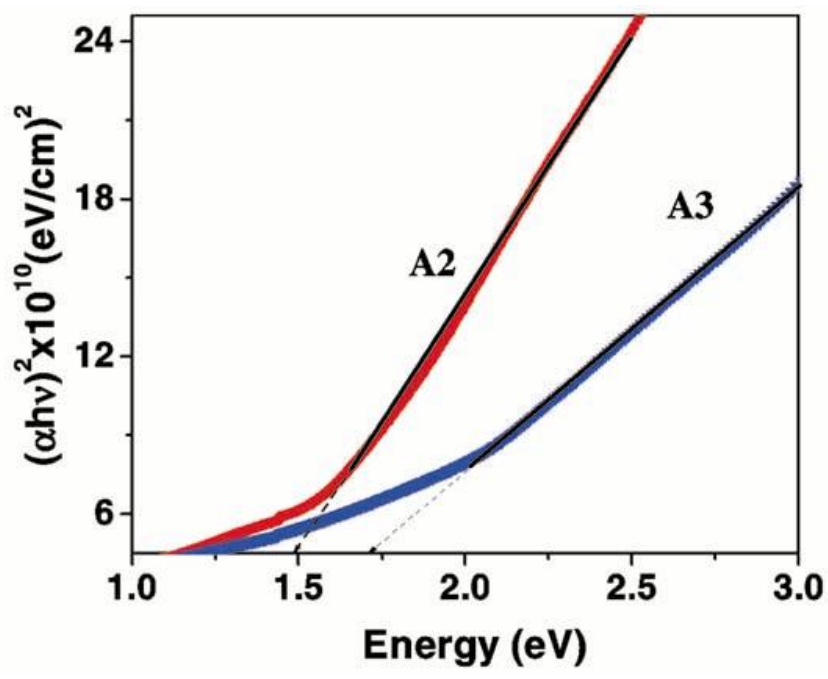

Figure 5. Plot of $(\alpha h v)^{2}$ vs $h v$ for samples $\mathrm{A} 2$ and $\mathrm{A} 3$.

XPS results which show that annealing in nitrogen plasma increases nitrogen content in the film. This excess nitrogen may be responsible for larger carrier concentration.

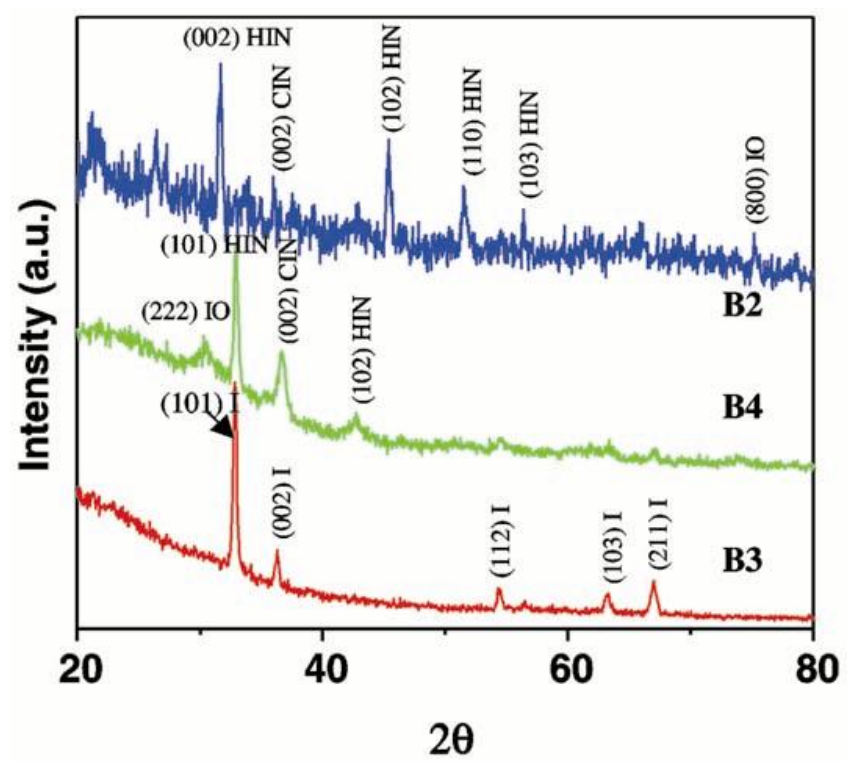

Figure 6. XRD of B2, B3 and B4 samples. The peaks corresponding to the tetragonal indium, cubic indium oxide, hexagonal indium nitride and cubic indium nitride have been indicated by appending ' $\mathrm{I}$ ', 'IO', ' $\mathrm{HIN}$ ' and ' $\mathrm{CIN}$ ' to the miller indices, respectively. 


\subsection{Plasma annealed In nanorod structures}

The present section discusses the effects of nitrogen plasma annealing of indium nanorods and thin films (samples B1, B2, B3 and B4). The results of the structural, optical and surface morphological measurements are presented and analysed.

3.2a Structural analysis: Figure 6 shows the XRD diffractograms of samples B2, B3 and B4, respectively. The XRD peaks have been indexed by comparing the observed ' $d$ ' values with the standard ' $d$ ' values in JCPDS data files. The $(h k l)$ indices of all the peaks have been marked in the figure. The sample B1 (In/AAM assembly) is a multiphase sample (XRD not shown here) consisting of indium, indium oxide and indium hydroxide phases. It must be mentioned that the XRD spectra of as obtained AAM (not shown here) indicates that it is amorphous in nature. The peaks in sample B3 correspond to the planes (101), (002), (112), (103) and (211) of tetragonal indium. The as-deposited In nanorods/AAM assembly (B1) and In thin film (B3) on being subjected to nitrogen plasma annealing for $5 \mathrm{~h}$ (sample B2) and $3 \mathrm{~h}$ (sample B4) show remarkable changes in the XRD spectra. In the XRD diffractogram of sample B2 new peaks corresponding to the planes (002), (102), (110) and (103) of hexagonal indium nitride and (002) plane of cubic indium nitride are seen. Similarly in sample B4 additional peaks corresponding to the planes (101) and (102) of hexagonal InN and (002) plane of cubic indium nitride are observed to appear. These additional peaks in the two samples match

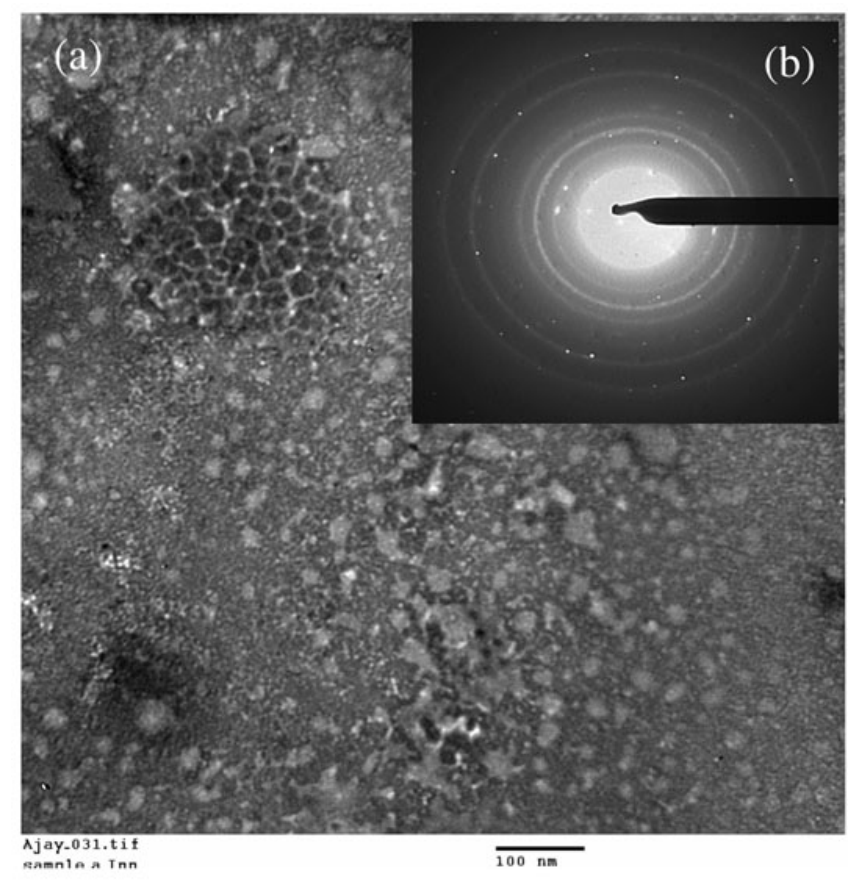

Figure 7. (a) TEM micrograph of sample B4 and (b) SAED pattern of sample B4. closely with the reported peaks for both hexagonal and cubic (Chai et al 2007; Nakamura et al 2007) forms of indium nitride. In samples B2 and B4, one additional peak corresponding to the (800) and (222) planes, respectively of indium oxide are also found. Hence, plasma annealing of starting indium nanorods and thin films converts them to indium nitride with both hexagonal and cubic forms coexisting. Thus N-plasma annealing is an effective technique to convert starting indium phase samples to indium nitride and at the same time stabilizing the metastable cubic phase.

Figure 7(a) shows TEM micrograph of sample B4 which is an indium thin film sample annealed in nitrogen plasma for $3 \mathrm{~h}$. The micrograph indicates uniform and complete coverage of the substrate by the film, with a broad size distribution of the particles. The average size of particles estimated from the TEM micrograph is 30$50 \mathrm{~nm}$. Figure 7(b) in the inset shows the selected area diffraction (SAD) of the sample. The ring pattern observed in the SAD pattern clearly reveals the polycrystalline nature of the film. The ' $d$ ' values have been estimated by using the SAD pattern of the gold film as a standard. It is found that ' $d$ ' values calculated from XRD and SAD match closely with the standard ' $d$ ' values for hexagonal
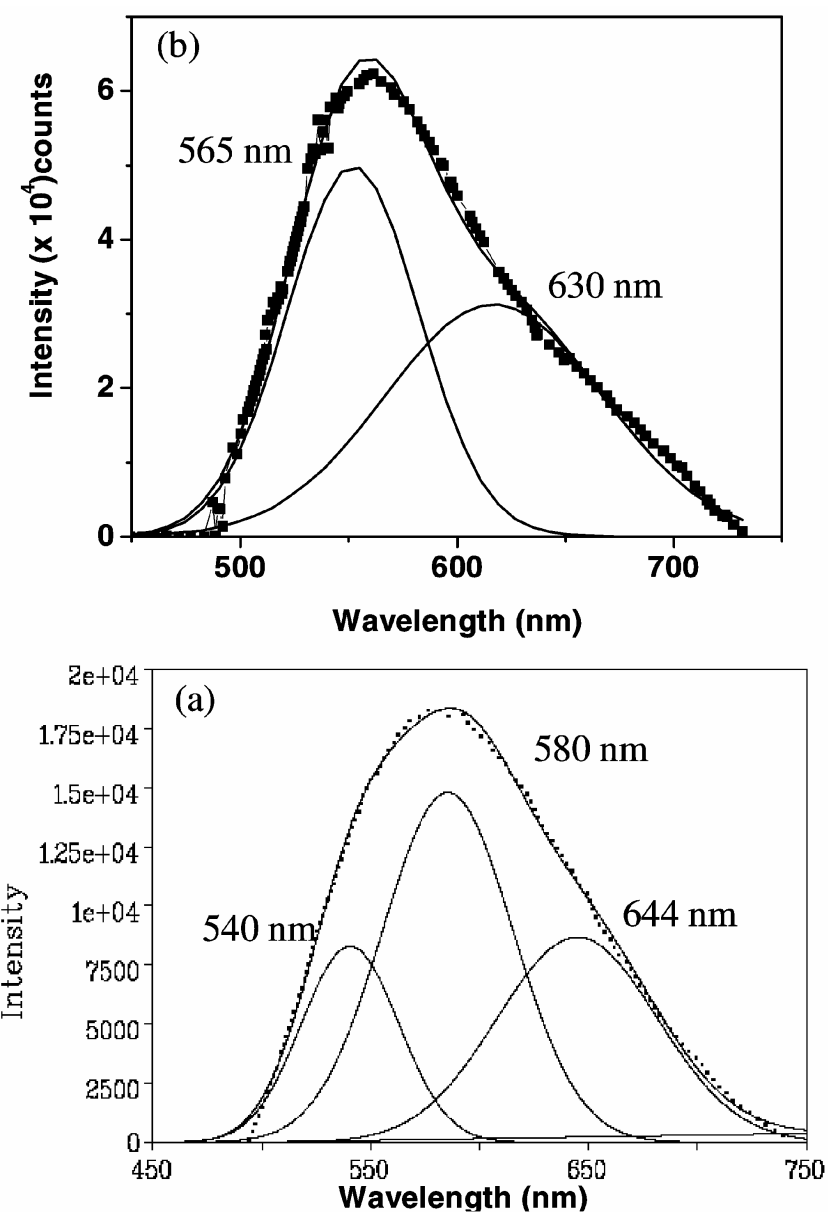

Figure 8. Photoluminescence spectra of (a) B2 and (b) B4. 


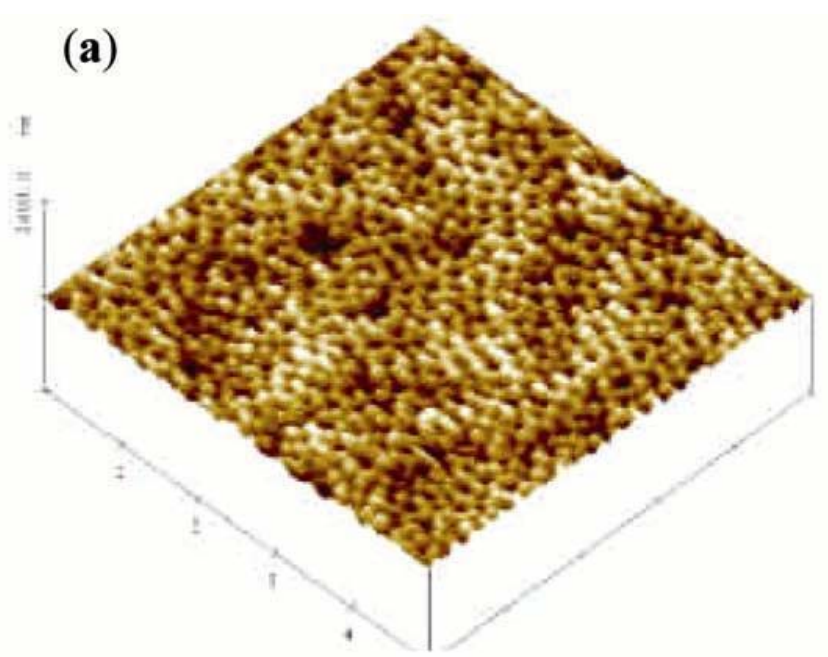

(b)

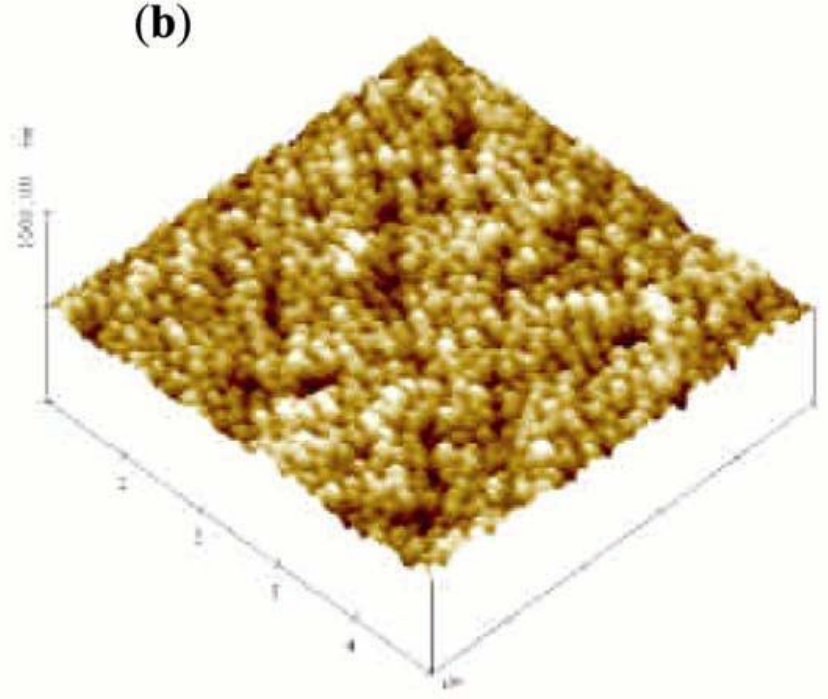

Figure 9. AFM images of (a) AAM and (b) B1.

and cubic InN. Thus SAD results further confirm that annealing indium films in nitrogen plasma converts them to indium nitride.

3.2b Optical absorption analysis: Figures 8(a) and (b) show the PL spectra of the samples B2 and B4, respectively. The peak fitting has been carried out by assuming gaussian line shapes. Both the samples exhibit broad emission peaks which have been deconvoluted into three peaks in sample B2 and two peaks in sample B4. The three peaks in sample B2 are centred at $540 \mathrm{~nm}, 585 \mathrm{~nm}$ and $644 \mathrm{~nm}$, corresponding to emission energies $2.29 \mathrm{eV}$, $2.12 \mathrm{eV}$ and $1.92 \mathrm{eV}$, respectively. On the other hand, the two peaks in sample B4 are centred at $565 \mathrm{~nm}$ and $630 \mathrm{~nm}$, corresponding to emission energies of $2.19 \mathrm{eV}$ and $1.97 \mathrm{eV}$, respectively. The emission energies in the two samples are in yellow red range of the electromagnetic spectrum. The lower energy emission (1.97 and $1.92 \mathrm{eV}$ ) in the two samples is close to the reported value for band edge luminescence in hexagonal $\mathrm{InN}$, while higher energy emission $(2 \cdot 19 \mathrm{eV}$ and $2 \cdot 12 \mathrm{eV})$ can be attributed to the band to band transition in cubic InN (Chai et al 2007). It must be mentioned that XRD of the two samples also shows the presence of the metastable cubic form of InN in the two samples in addition to the stable hexagonal form. This shows that nitrogen plasma annealing helps in synthesizing metastable cubic phase along with stable hexagonal phase. Sample B2 exhibits one additional peak at $540 \mathrm{~nm}$ which may be due to $\mathrm{In}_{2} \mathrm{O}_{3}$ phase in the sample and has been reported to occur as a result of deep level or trap state emissions resulting from oxygen deficiencies (Guha et al 2004).

It has been reported that the presence of hydrogen in the reaction chamber has a detrimental effect on the luminescence properties of InN and normally leads to quenching of the signal. It has already been shown that hydrogen in the growth chamber not only increases hydrogen related defects, but also decreases the deposition rate (Koukitu et al 1997). The present synthesis method avoids the use of ammonia, both, in the deposition of $\mathrm{InN}$ by ARE, and in the annealing of indium, or indium nitride films. Therefore, there are minimal chances of hydrogen related defects being present in the films. This may be the reason for observing a strong PL signal even at room temperature, which shows samples prepared are of good crystalline quality.

3.2c Surface morphological analysis: Figure 9(a) shows the three-dimensional AFM image $(5 \times 5 \mu \mathrm{m})$ of the top view of as obtained AAM which has been used to carry out the electrochemical deposition. It is clear from the image that the AAM has a porous structure with a pore size of around 150-200 $\mathrm{nm}$. The pores are separated by a distance of around $100-150 \mathrm{~nm}$. The three-dimensional AFM image $(5 \times 5 \mu \mathrm{m})$ of the top view of as deposited sample Bl after etching out a part of template surface is shown in figure 9(b). The pores visible in figure 9(a) are seen to be filled up in figure 9(b) and a comparatively smoother morphology is apparent. XRD analysis of B2, discussed previously, has already confirmed the formation of indium nitride. Thus the combined XRD and AFM/SEM analysis clearly establish that in the present study successful synthesis of $\mathrm{InN}$ nanorods inside the pores of AAM has been achieved by nitrogen plasma annealing of the indium nanorod structures.

\section{Conclusions}

InN nanocrystalline layers with pure wurtzite structure have been formed by using activated reactive evaporation method. On annealing in nitrogen plasma, the nitrogen content of the InN layers is found to increase leading to an increase in carrier concentration and hence the bandgap. Both crystallinity and conductivity of InN nanocrystalline layers also increase remarkably. The technique of 
nitrogen plasma annealing has also been employed to synthesize InN nanorod structures by a two-step process. In the first step, indium nanorod structures were synthesized by electrochemical means in the form of In/AAM assembly. The second step consisted of conversion of In nanorods to InN nanorods by annealing in nitrogen plasma. It is found that both cubic and hexagonal crystal structures of indium nitride are stabilized. The results of the present study show that nitrogen plasma annealing can be used in a variety of ways to improve the properties of InN layers or to convert In to $\mathrm{InN}$ in nanoparticle or nanorod form. The technique is advantageous as In nanostructures formed using different techniques can be converted to InN nanostructures at low temperatures.

\section{Acknowledgement}

This work has been done as a part of the Core Group on Nano Science and Technology of Department of Science and Technology (DST), Govt. of India.

\section{References}

Bhuiyan A G, Hashimoto A and Yamamoto A $2003 \mathrm{~J}$. Appl. Phys. 942779
Butcher K S A and Tansley T L 2005 Superlattices and Microstructures 381

Chai X M, Cheung K Y Djurišić and Xie M H 2007 Mater. Lett. 611563

Davydov Y Yu et al 2002 Phys. Status Solidi (b) 229 R1

Fu S P and Chen Y F 2004 Appl. Phys. Lett. 851523

Guha P, Kar S and Chaudhuri S 2004 Appl. Phys. Lett. 85 3851

Inushima T, Mamutin V V, Vekshin V A, Ivanov S V, Sakon T, Motokawa M and Ohoya S 2001 J. Cryst. Growth 227-228 481

Koukitu A, Takahashi N and Seki H 1997 Jpn J. Appl. Phys. 36 L1136

Mahboob I, Veal T D, McConville C F, Lu H and Schaff W J 2004 Phys. Rev. Lett. 92036804

Mann A K, Varandani D, Mehta B R, Malhotra L K and Shivaprasad S M 2005 J. Nanosci. Nanotechnol. 51858

Mann A K, Varandani D, Mehta B R and Malhotra L K 2007 J. Appl. Phys. 101084304

Nakamura T, Tokumoto Y, Katayama R, Yamamoto T and Onabe K 2007 J. Crystal Growth 301-302 508

Tansley T L and Foley C P 1986 J. Appl. Phys. 593241

Wu J et al 2002 Appl. Phys. Lett. 803967

Wu J et al 2004 Appl. Phys. Lett. 842805

Xiao J, Xie Y, Tang R and Luo W 2003 Inorg. Chem. 42107

Yodo T, Yona H, Ando H, Nosei D and Harada Y 2002 Appl. Phys. Lett. 80968 